\title{
Hemophilia Joint Health Score
}

National Cancer Institute

\section{Source}

National Cancer Institute. Hemophilia Joint Health Score. NCI Thesaurus. Code C159553.

A physiotherapist-administered assessment that measures the impact of recurrent bleeding on the elbows, knees, and ankles across 11 symptoms: swelling, duration of swelling, muscle atrophy, axial alignment, crepitus on motion, flexion loss, instability, joint pain, strength, and gait. Each of the 11 symptoms is rated on a Likert-style scale ranging from 1 to 4, with higher numbers indicating greater severity of symptoms. Scores from the individual items are added together to give a joint score, gait score, and a total score, with higher scores indicating greater joint damage. 